\title{
Neutrophil/lymphocyte ratio predicts chemotherapy outcomes in patients with advanced colorectal cancer
}

\author{
W Chua ${ }^{1,2}$, KA Charles ${ }^{3}$, VE Baracos ${ }^{4}$ and SJ Clarke B,, $2^{2}$ \\ 'Sydney Cancer Centre, Concord Repatriation General Hospital, Hospital Road, Concord, New South Wales 2139 , Australia; ${ }^{2}$ Faculty of Medicine, \\ University of Sydney, Sydney, New South Wales, Australia; ${ }^{3}$ School of Medical Sciences (Pharmacology) and Bosch Institute, University of Sydney, Sydney, \\ New South Wales, Australia; ${ }^{4}$ Department of Oncology, University of Alberta, Edmonton, Alberta, Canada
}

BACKGROUND: Advances in the treatment of metastatic colorectal cancer ( $\mathrm{mCRC}$ ) in the last decade have significantly improved survival; however, simple biomarkers to predict response or toxicity have not been identified, which are applicable to all community oncology settings worldwide. The use of inflammatory markers based on differential white-cell counts, such as the neutrophil/ lymphocyte ratio (NLR), may be simple and readily available biomarkers.

METHODS: Clinical information and baseline laboratory parameters were available for 349 patients, from two independent cohorts, with unresectable $\mathrm{mCRC}$ receiving first-line palliative chemotherapy. Associations between baseline prognostic variables, including inflammatory markers such as the NLR and tumour response, progression and survival were investigated.

RESULTS: In the training cohort, combination-agent chemotherapy $(P=0.00 \mathrm{I})$ and $\mathrm{NLR} \leqslant 5(P=0.003)$ were associated with improved clinical benefit. The ECOG performance status $\geqslant 1(P=0.002), N L R>5(P=0.01)$, hypoalbuminaemia $(P=0.03)$ and single-agent chemotherapy $(P<0.000 \mathrm{I})$ were associated with increased risk of progression. The ECOG performance status $\geqslant 1$ $(P=0.004)$ and NLR $>5(P=0.002)$ predicted worse overall survival $(O S)$. The NLR was confirmed to independently predict $O S$ in the validation cohort $(P<0.000 \mathrm{I})$. Normalisation of the NLR after one cycle of chemotherapy in a subset of patients resulted in improved progression-free survival $(P=0.012)$.

CONCLUSION: These results have highlighted NLR as a potentially useful clinical biomarker of systemic inflammatory response in predicting clinically meaningful outcomes in two independent cohorts. Results of this study have also confirmed the importance of a chronic systemic inflammatory response influencing clinical outcomes in patients with $\mathrm{mCRC}$.

British Journal of Cancer (20I I) I 04, I288-1295. doi:I0.1038/bjc.201 I.I00 www.bjcancer.com

Published online 29 March 201 I

(C) 201। Cancer Research UK

Keywords: colorectal cancer; prognosis; neutrophil/lymphocyte ratio; cancer-associated inflammation

Colorectal cancer (CRC) is the third leading cause of worldwide cancer mortality after lung and stomach cancer and is responsible for 639000 deaths or $1.1 \%$ of total deaths (World Health Organisation, 2004). There have been major advances in the treatment of metastatic CRC (mCRC) in the last 10-15 years, involving the introduction of new cytotoxic and molecular targeted therapies. However, use of these newer treatments result in increased toxicities and are prohibitively expensive. Hence, there is a need for accurate predictors of outcomes from treatment, in particular, in identifying those patients who are more likely to benefit by being assisted in rationalising increasingly expensive treatments, especially in under-resourced communities.

Tumour development and growth occurs as a result of interactions among the tumour, host-derived stromal tissues including blood vessels and host immune/inflammatory cells (see Figure 1), with chronic inflammation having an important role in cancer development and progression (Balkwill and Mantovani, 2010; Coussens and Werb, 2002). Lymphocytic infiltration in

*Correspondence: Dr SJ Clarke; E-mail: stephen.clarke@sydney.edu.au Received I December 2010; revised I5 February 2011; accepted 25 February 201 I; published online 29 March 2011 primary colorectal tumour tissue with different lymphocyte subpopulations has been investigated as potential prognostic factors (Pagès et al, 2005; Galon et al, 2006). This chronic inflammatory state also has effects on normal tissues, including the liver, resulting in an ongoing release of 'acute-phase proteins' that may be used to monitor this process. Current prognostication in advanced CRC, as in other malignancies, involves a poorly defined combination of clinical experience with the use of relatively crude and subjective covariates, such as performance status, with few markers in clinical practice apart from the use of $k$-ras mutation status and treatment with epidermal growth-factor receptor inhibitors (Bokemeyer et al, 2009; Koopman et al, 2009; Van Cutsem et al, 2009; Chua et al, 2010).

Over the last 10 years, laboratory markers of a systemic inflammatory response, including plasma C-reactive protein concentration (CRP), hypoalbuminaemia and Glasgow Prognostic Score (GPS, which combines CRP and albumin), and absolute white cell or its components (neutrophils, neutrophil/lymphocyte ratios (NLRs) and platelet/lymphocyte ratios (PLRs)) have been investigated as prognostic and predictive markers in different cancer populations, with the best evidence for their use demonstrated in surgical patients with CRC (Roxburgh and McMillan, 2010). Emerging evidence suggests that elevated baseline levels of 


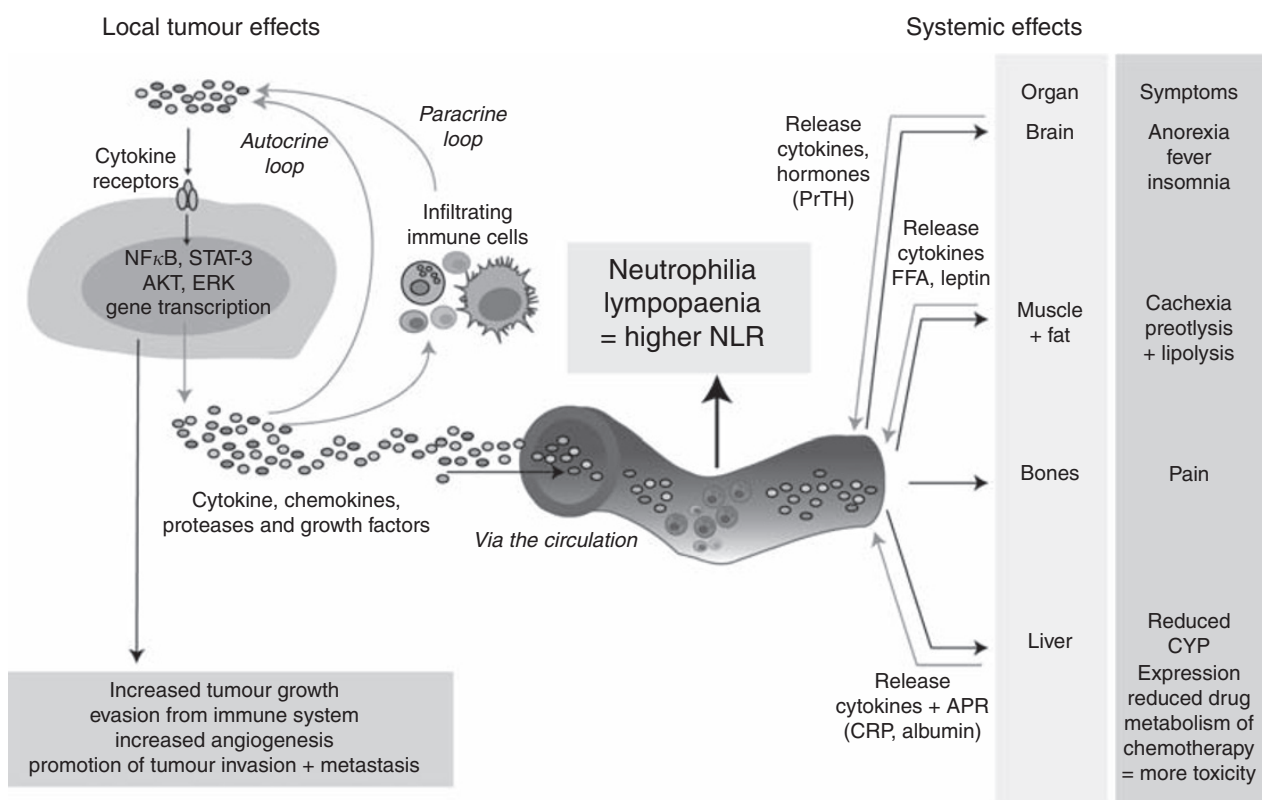

Figure I The effect of cytokines in the local tumour environment and systemic organs, and the clinical manifestations of these interactions. Red arrows indicate cytokines being released from either tumour or other organ.

CRP (Hilmy et al, 2005; Crumley et al, 2006a; Mitsuru et al, 2009; Johnson et al, 2010), abnormal GPS scores (Forrest et al, 2003; Crumley et al, 2006b; McMillan, 2009) and elevated NLR (Yamanaka et al, 2007; Halazun et al, 2008, 2009; Liu et al, 2010) or PLR (Smith et al, 2009) are not only negatively correlated with outcome after surgical resection but also in those with inoperable cancers. These inflammation scores based on readily available and inexpensive tests could potentially be ideal biomarkers of outcome in patients with mCRC.

Evidence for the use of these inflammatory markers as direct predictors of outcome in patients with advanced malignancy receiving first-line chemotherapy is lacking. Two recent studies have highlighted the use of the systemic inflammatory response in predicting survival (Teramukai et al, 2009) and toxicity (Arieta et al, 2010) in patients receiving chemotherapy for advanced nonsmall-cell lung cancer. Neutrophilia has been shown to be an adverse prognostic factor in patients receiving first-line oxaliplatin-based chemotherapy for CRC (Michael et al, 2006). An elevated NLR in CRC patients with liver-only colorectal metastases receiving neoadjuvant chemotherapy before surgical resection of liver metastases predicted worse survival (Kishi et al, 2009). In addition, those patients in whom NLR normalised after chemotherapy had significantly improved 1-, 3- and 5-year survival similar to patients with NLR $\leqslant 5$ at baseline. These data suggest that NLR may be a readily available and useful biomarker for monitoring early response and prognosis with chemotherapy for CRC (Kishi et al, 2009). The aims of the current study were to investigate (1) NLR in predicting treatment response, toxicity and survival in medical patients receiving first-line chemotherapy for advanced CRC in a training set; (2) validating the results from the training cohort in a separate community patient cohort; and (3) assessing the impact of normalisation of NLR for monitoring early response during chemotherapy.

\section{PATIENTS AND METHODS}

\section{Study population}

In total, there were 349 patients with available clinical information and baseline laboratory parameters. The training set consisted of
171 patients enrolled in first-line chemotherapy trials at the Sydney Cancer Centre for advanced CRC between 1999 and 2007. The independent validation set included 178 patients from a community-based clinical database in the province of Alberta and included patients referred to medial oncology units at the Cross Cancer Institute who received first-line chemotherapy for advanced CRC between 2004 and 2007 (Prado et al, 2008). Table 1 lists the comparative baseline clinical information and laboratory parameters for both cohorts before chemotherapy commencement.

\section{Methods}

Baseline clinical information and biochemical evaluation, including full blood count (neutrophils, lymphocytes, haemoglobin and platelets) and albumin before chemotherapy commencement, were collected in a database for patients in both the training and validation sets. Alkaline phosphatase was also collected in the training set. Prognostic variables with $>10 \%$ missing data were not included in the analysis. Differential white-cell counts (neutrophils and lymphocytes) were also collected for patients before cycle 2 of chemotherapy. Response rates, dates of progression and survival were available for patients in the training set; however, only survival data were available for patients in the validation cohort. Dates of death were followed up by the investigators through hospital records, local Cancer Registries or phone contact through patient relatives, local medical practitioners and palliative-care services. Patients were consented to undergo analyses before commencing chemotherapy, and the study was approved by institutional research ethics committees in both Sydney and Edmonton.

\section{Statistical analysis}

Statistical analyses were performed using SPSS Graduate Version 17.0 (IBM Corporation 2010, Somers, NY, USA). Response rates were determined according to criteria determined by individual clinical trials, RECIST criteria. Clinical response was defined as either complete or partial response and non-response as either stable or progressive disease. Clinical benefit was defined as complete response, partial response and stable disease and no benefit as progressive disease alone. Progression-free survival 
Table I Baseline patient characteristics in the training and validation sets before commencement of chemotherapy

\begin{tabular}{|c|c|c|c|}
\hline & $\begin{array}{c}\text { Training set } \\
(n=17 I) \\
\text { No. (\%) }\end{array}$ & $\begin{array}{c}\text { Validation set } \\
\qquad(n=I 78) \\
\text { No. }(\%)\end{array}$ & $P$-value \\
\hline Age, median (range) & $61(33-84)$ & $63(32-85)$ & 0.40 \\
\hline Gender $(\mathrm{M} / \mathrm{F})$ & $|10 / 6|(64 / 36)$ & $100 / 73(56 / 44)$ & 0.22 \\
\hline Primary cancer site & $-^{\mathrm{a}}$ & & \\
\hline Colon & $103(64)$ & $116(65)$ & \\
\hline Rectosigmoid & $52(32)$ & $23(13)$ & \\
\hline Rectum & $4(3)$ & $39(22)$ & \\
\hline Synchronous & $2(1)$ & 0 & $<0.000$ \\
\hline \multicolumn{4}{|l|}{ ECOG performance status } \\
\hline 0 & $85(50)$ & $30(17)$ & \\
\hline । & $81(47)$ & $96(54)$ & \\
\hline$\geqslant 2$ & $5(3)$ & $52(29)$ & $<0.000$ I \\
\hline \multicolumn{4}{|l|}{ Chemotherapy regimen } \\
\hline Single agent only & $43(25)$ & $34(19)$ & \\
\hline $\begin{array}{l}\text { Combination } \\
\text { chemotherapy } \pm \text { biologicals }\end{array}$ & $128(75)$ & $92(52)$ & \\
\hline Unknown & 0 & $52(29)$ & $<0.0001$ \\
\hline \multicolumn{4}{|l|}{ Number of sites } \\
\hline 1 & $91(53)$ & NA & - \\
\hline$>1$ & $80(47)$ & & \\
\hline \multicolumn{4}{|l|}{ Baseline levels of prognostic factors } \\
\hline Albumin, median (range), $\mathrm{gl}^{-1}$ & $40(27-48)$ & $39(20-47)$ & \\
\hline $\begin{array}{l}\text { Carcinoembryonic antigen, } \\
\text { median (range) }\end{array}$ & $38.6(0.7-945)$ & NA & \\
\hline Haemoglobin, median (range) & $127(82-162)$ & $122(78-170)$ & \\
\hline Neutrophils, median (range) & $5.5(1.5-14.8)$ & $5.1(1.9-21.4)$ & \\
\hline Lymphocytes, median (range) & $1.4(0.4-4.9)$ & $1.5(0.2-3.3)$ & \\
\hline $\begin{array}{l}\text { Neutrophil-lymphocyte ratio, } \\
\text { median (range) }\end{array}$ & $3.7(1.0-30.8)$ & $3.5(0.9-74.5)$ & \\
\hline$\leqslant 5$ & $120(7 \mid)$ & $123(69)$ & \\
\hline$>5$ & $49(29)$ & $55(31)$ & \\
\hline
\end{tabular}

Abbreviations: $E C O G=$ Eastern Cooperative Oncology Group; $F=$ female $\mathrm{M}=$ male; $\mathrm{NA}=$ not available. ${ }^{\mathrm{a}}$ Missing data for 10 patients (training set).

(PFS) was defined as the date of commencing protocol treatment to the date of first progression or death from any cause without progression. Overall survival (OS) was defined as the date from the date of commencing protocol treatment to the date of death from any cause. The $\chi^{2}$-tests were used to test associations between variables of interest (grouped using standard thresholds) and clinical response or benefit. Multivariate modelling was used for calculation of hazard ratios and clinical response and benefit. The follow-up period commenced at the start of chemotherapy with the censor date of January 2010. Survival analysis was performed using the Kaplan-Meier method with log-rank test in univariate analyses. Cox regression analysis was used for multivariate survival analysis and for calculation of hazard ratios.

\section{RESULTS}

\section{Patient characteristics}

Baseline clinical demographics and laboratory values for both training and validation sets are presented in Table 1 . There were no differences in age and gender between the two cohorts. However, a significantly higher proportion of patients in the validation cohort had rectal cancer as the primary tumour site and had ECOG $P S \geqslant 1$. The majority of patients in both cohorts received combination chemotherapy \pm a biological agent.

\section{Prognostic variables in training set}

Table 2 shows the univariate analyses between prognostic variables of interest and clinical benefit, PFS and OS in the training set. At the time of analysis, all patients had progressed on chemotherapy and 169 patients were deceased. The overall clinical response (complete response and partial response) was 55\% (93 out of 168 evaluable patients) and clinical benefit (complete response, partial response and stable disease) was 75\% (128 out of 168 evaluable patients). The median PFS was 6.7 months (95\% CI 5.6-7.8 months) and OS was 15.3 months (95\% CI 12.4-18.2).

Clinical benefit and response Younger age ( $\leqslant 65$ years old), ECOG performance status 0 , absence of hypoalbuminaemia, normal alkaline phosphatase, low or normal neutrophil counts and NLR $\leqslant 5$ were associated with improved clinical benefit (Table 2). Similarly, younger age ( $\leqslant 65$ years old), ECOG performance status 0 and NLR $\leqslant 5$ were associated with improved clinical response. In addition, combination-agent chemotherapy was also associated with improved clinical response.

PFS and OS Variables predicting improved PFS included younger age, ECOG performance status 0 , combination-agent chemotherapy, single site of metastasis, absence of neutrophilia or hypoalbuminaemia and NLR $\leqslant 5$ (Table 2 and Figure $2 \mathrm{~A}$ ). The following variables were associated with improved OS: younger age, ECOG PS 0, combination-agent chemotherapy, absence of neutrophilia or anaemia. Hypoalbuminaemia, elevated alkaline phosphatase and NLR $>5$ were also significantly associated with worse OS (Table 2 and Figure 2B).

Multivariate analysis In multivariate analysis performed in the training set (Table 3), combination-agent chemotherapy and NLR $\leqslant 5$ were associated with improved clinical benefit. The ECOG performance status $\geqslant 1$, NLR $>5$, hypoalbuminaemia and singleagent chemotherapy were associated with increased risk of progression. The ECOG performance status $\geqslant 1$ and NLR $>5$ predicted worse OS.

Prognostic variables according to NLR Table 4 summarises analysis of baseline characteristics and prognostic variables according to NLR groups. Patients with NLR $>5$ were more likely to suffer from hypoalbuminaemia $(P$-level $<0.0001)$ and elevated alkaline phosphatase $(P$-level 0.008$)$. The association between NLR and gender $(P$-level 0.06$)$ and number of metastatic sites $(P$-level $0.05)$ approached statistical significance.

\section{NLR in validation cohort}

At the time of analysis, $82 \%$ (146 out of 178) of patients were deceased. The median OS in this cohort was 16.8 months $(95 \% \mathrm{CI}$ 13.1-20.4 months). Independent predictors of survival from the training cohort (ECOG performance status and NLR) were tested in the validation cohort. The NLR was statistically significantly associated with OS $(P$-level $<0.0001)$. Patients with NLR $\leqslant 5$ had median OS of 19.1 months (95\% CI 15.3-22.8) compared with patients with NLR $>5$ (median OS 11.3 months; 95\%CI 8.3-14.3; Figure $2 \mathrm{C}$ ). The ECOG performance status was not predictive of survival in this cohort (median OS for ECOG 0 was 21.5 months (95\% CI 4.1-38.9) and PS $\geqslant 115.7$ months (95\% CI 13.1-18.3; P-level 0.15)).

\section{Normalisation of NLR pre-cycle 2 and correlation with PFS and OS (training cohort)}

Patients were categorised into the following categories: (1) patients with $\mathrm{NLR} \leqslant 5$ at baseline $(n=120$; cohort 1$)$, (2) NLR $>5$ at baseline and before cycle 2 of chemotherapy $(n=21$; cohort 2$)$ and 
Table 2 Univariate analysis of clinicopathological factors, inflammatory response and response rate, PFS and OS in training set

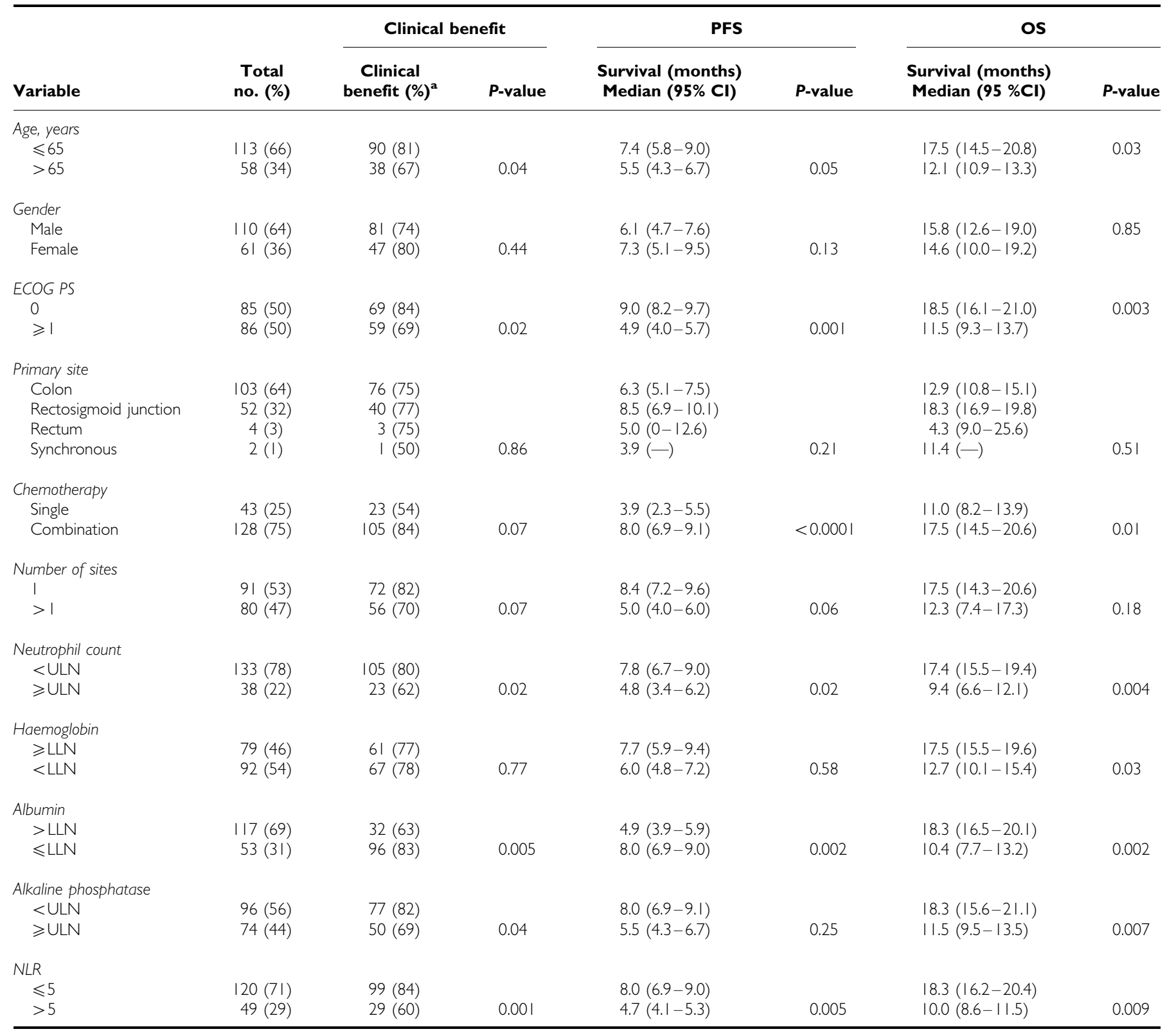

Abbreviations: $\mathrm{Cl}=$ confidence interval; $\mathrm{ECOG}=$ Eastern Cooperative Oncology Group; OS = overall survival; LLN= lower limits of normal; NLR=neutrophil/lymphocyte ratio; PFS = progression-free survival; PS = performance status; ULN = upper limits of normal. ${ }^{a}$ Missing data $(<10 \%)$ for some prognostic variables.

(3) NLR $>5$ at baseline with normalisation of NLR $\leqslant 5$ before cycle 2 of chemotherapy $(n=21$; cohort 3$)$. Patients with normalisation of NLR before cycle 2 of chemotherapy (cohort 3 ) had an improved PFS of 5.8 months (95\% CI 4.1-7.5) compared with patients without normalisation of NLR pre-cycle 2 (cohort 2; median PFS 3.7 months; 95\% CI 0.6-6.8 months; P-level 0.012; Figure 3A). Normalisation of NLR improved median OS from 9.4 months (cohort 2; 95\% CI 3.2-15.5) to 12.1 months (cohort 3; 95\% CI $7.3-16.8$ ) in patients with a persistently elevated NLR, although this did not reach statistical significance ( $P$-level 0.053; Figure $3 \mathrm{~B}$ ). Patients with normalised NLR before cycle 2 of chemotherapy (cohort 3) did not have significantly different median PFS (5.8 months (95\% CI $4.1-7.5)$ vs 8.0 months (95\% CI 6.9-9.0); $P$-level 0.37$)$ or OS (12.1 months (95\% CI 7.3-16.8) vs 18.3 months (95\% CI 16.2-20.4); P-level 0.77) in comparison with patients with NLR $\leqslant 5$ before chemotherapy commencement (cohort 1 ; Figures $3 \mathrm{~A}$ and $\mathrm{B}$ ). Normalisation of NLR before cycle 2 of chemotherapy was not performed in the validation cohort, as there was $>10 \%$ of missing data for this patient group.

\section{DISCUSSION}

This is the first study, to our knowledge, to describe the use of NLR in a non-selected unresectable mCRC setting for patients receiving first-line palliative chemotherapy to provide useful information regarding prognostication, and the data have been validated in an independent community-based cohort. These results support the use of NLR as a marker of systemic inflammatory response and as an independent predictor of clinical benefit, progression and survival in patients receiving chemotherapy for mCRC. An NLR cutoff $>5$ was able to identify a subset of patients least likely to respond to chemotherapy ( $40 v s 16 \%)$ and those at higher risk of progression and death (HR 1.6 and 1.7, respectively). A cutoff 

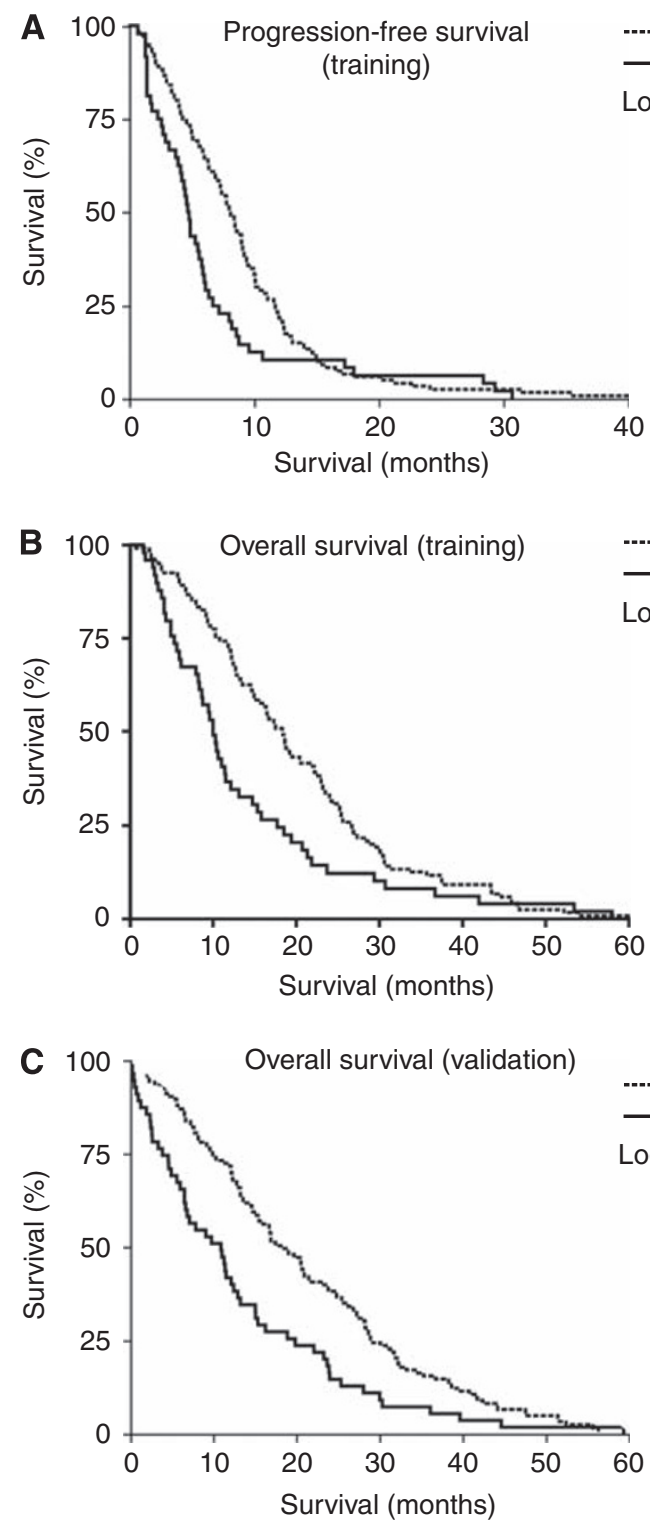

Figure 2 PFS according to NLR in $(\mathbf{A})$ training cohort. OS according to $N L R$ in $\mathbf{B}$ (training) and $(\mathbf{C})$ validation cohorts of patients with $\mathrm{mCRC}$ treated with chemotherapy.

score of 5 was chosen on the basis of previous studies (Halazun et al, 2008, 2009; Kishi et al, 2009) and this represents a simple measurement to use in clinical practice, although other cutoffs have been used (Duffy et al, 2006; Cho et al, 2009). This identifies $\sim 30 \%$ of CRC patients with a raised NLR receiving first-line chemotherapy in both cohorts and associated with shorter survival of up to 8 months. These results are highly clinically relevant in this increasingly common malignancy.

In addition, evidence for significantly improved outcomes with normalisation of NLR after the first cycle is promising for possible manipulation of the systemic inflammatory response through targeted anti-inflammatory mediators such as IL-6 blocking antibodies. If the use of NLR and normalistion of NLR after cycle 1 are confirmed, this would provide additional prognostic information for clinicians at an earlier time point before conventional staging with computed tomography scans and potentially identify a proportion of patients in whom further treatment may be futile. For example, in the training cohort, there was NLR normalisation after one cycle of chemotherapy in $50 \%$ (21 out of 42) of evaluable patients, which resulted in a 2-month PFS
Table 3 Multivariate analysis of prognostic factors of clinical response, PFS and OS (training set)

\begin{tabular}{|c|c|c|c|}
\hline & Prognostic variable & Hazard ratio $(95 \% \mathrm{Cl})$ & P-level \\
\hline \multirow[t]{6}{*}{ Clinical benefit } & Chemotherapy type & & \\
\hline & Single agent & 1 & \\
\hline & Combination & $4.0(1.8-9.2)$ & 0.001 \\
\hline & $N L R$ & & \\
\hline & $>5$ & I & \\
\hline & $\leqslant 5$ & $3.4(1.5-7.8)$ & 0.003 \\
\hline \multirow[t]{12}{*}{ PFS } & ECOG performance status & & \\
\hline & 0 & 1 & \\
\hline & I or more & $1.5(1.1-2.1)$ & 0.02 \\
\hline & NLR & & \\
\hline & $\leqslant 5$ & I & \\
\hline & $>5$ & $1.6(1.1-2.2)$ & 0.01 \\
\hline & Albumin & & \\
\hline & $\geqslant L L N$ & I & \\
\hline & $<\mathrm{LLN}$ & $1.5(1.0-2.1)$ & 0.03 \\
\hline & Chemotherapy type & & \\
\hline & Combination & I & \\
\hline & Single agent & $2.8(1.9-4.0)$ & $<0.0001$ \\
\hline \multirow[t]{6}{*}{ OS } & ECOG performance status & & \\
\hline & 0 & 1 & \\
\hline & I or more & $1.6(1.2-2.2)$ & 0.004 \\
\hline & $N L R$ & & \\
\hline & $\leqslant 5$ & I & \\
\hline & $>5$ & $1.7(1.2-2.5)$ & 0.002 \\
\hline
\end{tabular}

Abbreviations: $\mathrm{Cl}=$ confidence interval; $\mathrm{ECOG}=$ Eastern Cooperative Oncology Group; $O S=$ overall survival; $L L N=$ lower limits of normal; $N L R=$ neutrophil/ lymphocyte ratio; PFS = progression-free survival.

improvement (5.8 vs 3.7 months) compared with patients without NLR normalisation. These data will permit not only retrospective evaluations of established large cohorts with known outcome data to corroborate these findings but also to undertake correlation with molecular characteristics, such as microsatellite instability and B-raf mutations, which are associated with worse cancer outcomes.

The strengths in our training cohort were that patient data were retrospectively analysed from robust prospectively collected data through entry into clinical trials. As the patients were eligible for enrolment in a clinical trial, it is highly unlikely that the elevated NLR was due to other active inflammatory diseases or infection or were requiring high doses of steroids; however, these issues should be specifically assessed in future studies. Other independent predictive variables identified from the training cohort, such as performance status, use of combination chemotherapy and hypoalbuminaemia, have also been reported from previous studies and strengthens the case for this cohort being representative of a palliative mCRC population. The median OS in both cohorts (15.3 and 16.8 months in training and validation cohorts, respectively) are shorter than those reported using modern combination chemotherapy regimens, which have median OS upwards of 24 months. However, a significant proportion of the patients in both cohorts received single-agent chemotherapy, with patients enrolled in chemotherapy trials from as early as 1999. There were also significant baseline differences in the types of chemotherapy regimens between the Australian and Canadian cohorts. In the Canadian cohort, up to $29 \%$ of patients did not have the type of chemotherapy specified, which may account for some of the survival difference between the two cohorts. The validation cohort in this study failed to identify performance status as an 
Table 4 Baseline characteristics according to NLR

\begin{tabular}{|c|c|c|c|}
\hline Variable & $\mathbf{N L R} \leqslant 5$ & NLR $>5$ & P-level \\
\hline Age (median) & 61 & 61 & 0.32 \\
\hline \multicolumn{4}{|l|}{ Gender } \\
\hline Male & $72(60 \%)$ & $37(76 \%)$ & \\
\hline Female & $48(40 \%)$ & $12(24 \%)$ & 0.06 \\
\hline \multicolumn{4}{|l|}{ ECOG performance status } \\
\hline 0 & $69(58 \%)$ & $24(49 \%)$ & \\
\hline 1 and 2 & $60(50 \%)$ & $25(51 \%)$ & 0.90 \\
\hline \multicolumn{4}{|l|}{ Number of metastatic sites } \\
\hline I & $69(58 \%)$ & $20(4 \mid \%)$ & \\
\hline$>1$ & $51(42 \%)$ & $29(59 \%)$ & 0.05 \\
\hline \multicolumn{4}{|l|}{ Primary site } \\
\hline Colon & $70(62 \%)$ & $31(67 \%)$ & \\
\hline Rectum and rectosigmoid junction & $42(37 \%)$ & $14(30 \%)$ & \\
\hline Synchronous & I (I\%) & I (1\%) & 0.61 \\
\hline \multicolumn{4}{|l|}{ Site of metastases } \\
\hline Liver & $96(80 \%)$ & $44(90 \%)$ & 0.13 \\
\hline Lung & $37(31 \%)$ & $17(35 \%)$ & 0.63 \\
\hline Other & $43(36 \%)$ & $21(43 \%)$ & 0.39 \\
\hline \multicolumn{4}{|l|}{ Anaemia } \\
\hline Present & $64(53 \%)$ & $28(57 \%)$ & \\
\hline Absent & $56(47 \%)$ & $21(43 \%)$ & 0.65 \\
\hline \multicolumn{4}{|l|}{ Hypoalbuminaemia } \\
\hline Present & $26(22 \%)$ & $26(53 \%)$ & \\
\hline Absent & $93(78 \%)$ & $23(47 \%)$ & $<0.000$ \\
\hline \multicolumn{4}{|l|}{ Alkaline phosphatase levels } \\
\hline$<U L N$ & 75 (63\%) & $20(41 \%)$ & \\
\hline$\geqslant U L N$ & 44 (37\%) & $29(59 \%)$ & 0.008 \\
\hline
\end{tabular}

Abbreviations: $\mathrm{ECOG}=$ Eastern Cooperative Oncology Group; NLR= neutrophil/ lymphocyte ratio; ULN = upper limits of normal.

independent prognosticator, which, although surprising, may reflect the community-based origins of this group. However, in both cohorts, the proportion of patients with NLR $>5$ was surprisingly consistent between the two cohorts (29 and 31\%). In spite of differences between the cohorts, NLR remained an independent prognosticator and may reflect that it is an even more robust and accurate prognosticator than performance status alone. The heterogeneity of treatment regimens used could be criticised; however, this is probably more reflective of day-to-day clinical practice.

The NLR is a simple, readily available and robust laboratory variable. Other authors have advocated the use of GPS or a modified GPS, based on albumin and CRP levels, and validated its use as a prognostic variable particularly in the pre-operative setting. Two studies have reported the use of GPS in patients receiving chemotherapy for mCRC and gastro-oesophageal malignancies (Crumley et al, 2008; Ishizuka et al, 2009). However, this assessment is complicated by the requirement for an additional blood test to measure CRP levels, which may not be readily available as was in the case of both our training and validation sets. The NLR, as a continuous variable, may also be a more accurate and dynamic variable reflecting acute changes in the inflammatory state of a patient rather than GPS, which is applied as a static, categorical variable. The NLR and GPS have not been compared in the same population in CRC patients, and this comparison should be undertaken to discern whether these two indices are overlapping or additive as indicators of cancer-associated inflammation. In CRC, the use of NLR has previously been confirmed as an independent prognostic factor in a cohort of patients with liveronly colorectal metastases, the majority of whom proceeded to
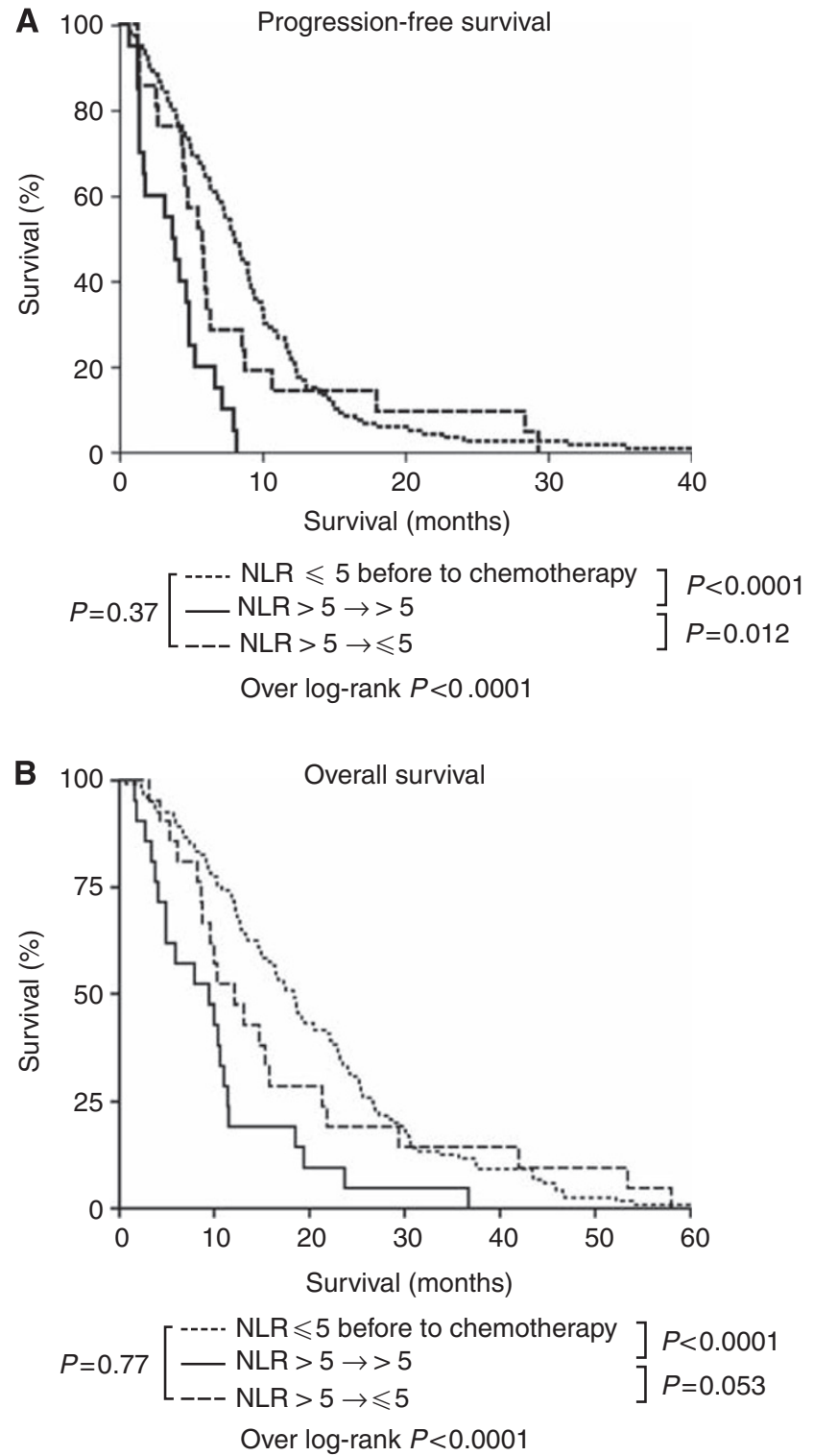

Figure 3 Changes in PFS $(\mathbf{A})$ and OS $(\mathbf{B})$ with normalisation of NLR in training cohort of $\mathrm{mCRC}$ treated with chemotherapy.

hepatic resection post chemotherapy (Kishi et al, 2009). Although this is an important subset of patients with mCRC, these patients would have been highly selected for surgical intervention and not representative of the majority of patients with mCRC. The findings in our study are not only consistent with this earlier report but also supports the use of NLR in a more generalised patient population receiving first-line chemotherapy both in a clinical trial and community setting. Although elevated NLR was correlated with the presence of hypoalbuminaemia and elevated alkaline phosphatase in this study (Table 4), other prognostic variables, such as performance status, site or extent of disease, were relatively wellbalanced between the high- and low-NLR groups, suggesting that NLR provides additional information than these other variables. The association of both raised NLR and hypoalbuminaemia is likely because of its role as a marker of systemic inflammation. The reasons for the correlation between alkaline phosphatase and NLR are unclear. Alkaline phosphatase may be a more accurate marker of the extent of liver involvement or indirectly related to systemic inflammation. The NLR has also been previously shown to independently predict outcomes in non-malignant disease, such 
as post-ST-segment elevation myocardial infarction (Núñez et al, 2008) and percutaneous coronary intervention (Duffy et al, 2006) in which the systemic inflammation response has been implicated as a major contributing factor. This adds credibility for the use of NLR as a potential biomarker of the systemic inflammatory response.

In recent years, there have been significant developments and discoveries in cancer genomics. The development of geneexpression-based arrays or examining germline single-nucleotide polymorphisms for defining prognosis or predicting response to therapy has limited clinical application even in the two most common malignancies, lung and breast cancers (Hartman et al, 2010; Subramanian and Simon, 2010). For example, Wacholder et al (2010) discovered that the inclusion of 10 common breast cancer genetic variants only modestly improved the performance of existing risk-assessment models in >11 000 patients, with little change in the predicted breast-cancer risk among most women, using currently available genetic information. These tests are also expensive and confined to use in developed countries, with limited application in under-resourced communities. A useful biomarker needs to be not only accurate and reproducible but also easily accessible. The prognostic importance of the systemic reaction to tumours has been relatively ignored in the quest for tumour-based molecular assessments of outcome. These data will encourage a re-evaluation of that approach.

These results have highlighted the use of a potential clinical biomarker of systemic inflammatory response in predicting clinically meaningful outcomes in two independent cohorts. In addition, results of the study have also confirmed the importance of a chronic systemic inflammatory response influencing clinical outcomes in patients with mCRC. Validation of these results in larger patient populations will allow many potential applications in the treatment of mCRC, a major cause of morbidity worldwide. Clinical applications include (1) prognostication and in-patient stratification in clinical trials, (2) as a marker of response to chemotherapy treatment and, more excitingly, (3) in identifying patients for possible interventions with anti-inflammatory mediators. The results of this study, we believe, strongly support the use of NLR in these settings, and more importantly, as a dynamic marker of interactions among tumour, host and the systemic inflammatory response.

\section{ACKNOWLEDGEMENTS}

We thank Jenny Peat for assistance with statistical analysis. Wei Chua was supported by a NSW Cancer Institute Fellowship (Australia) and a Pfizer Australia Cancer Research Grant. The research was facilitated by a Translational Colorectal Cancer Research Grant from the NSW Cancer Institute. This study was funded by the Canadian Institute of Health Research.

\section{Conflict of interest}

The authors declare no conflict of interest.

\section{REFERENCES}

Arieta O, Michel Ortega RM, Villaneuva-Rodriguez G, Serna-Thome MG, Flores-Estrada D, Diaz-Romero C, Rodriguez CM, Martinez L, SanchezLara K (2010) Association of nutritional status and serum albumin levels with development of toxicity in patients with advanced non-small cell lung cancer treated with paclitaxel-cisplatin chemotherapy: a prospective study. BMC Cancer 10: 50

Balkwill F, Mantovani A (2010) Cancer and inflammation: implications for pharmacology and therapeutics. Clin Pharmacol Ther 87: 401-406

Bokemeyer C, Bondarenko I, Makhson A, Hartmann JT, Aparicio J, de Braud F, Donea S, Ludwig H, Schuch G, Stroh C, Loos AH, Zubel A, Koralewski P (2009) Fluorouracil, leucovorin, and oxaliplatin with and without cetuximab in the first-line treatment of metastatic colorectal cancer. J Clin Oncol 27: 663-671

Cho H, Hur HW, Kim SW, Kim SH, Kim JH, Kim YT, Lee K (2009) Pretreatment neutrophil to lymphocyte ratio is elevated in epithelial ovarian cancer and predicts survival after treatment. Cancer Immunol Immunother 58: $15-23$

Chua W, Kho PS, Moore MM, Charles KA, Clarke SJ (2010) Clinical, laboratory and molecular factors predicting chemotherapy efficacy and toxicity in colorectal cancer. Crit Rev Oncol Hematol [E-pub ahead of print 16 August 2010; doi:10.101.6/j.critrevonc.2010.07.012]

Coussens LM, Werb Z (2002) Inflammation and cancer. Nature 420: $860-867$

Crumley AB, McMillan DC, McKernan M, Going JJ, Shearer CJ, Stuart RC (2006a) An elevated C-reactive protein concentration, prior to surgery, predicts poor cancer-specific survival in patients undergoing resection for gastro-oesophageal cancer. Br J Cancer 94: 1568-1571

Crumley ABC, McMillan DC, McKernan M, McDonald AC, Stuart RC (2006b) Evaluation of an inflammation-based prognostic score in patients with inoperable gastro-oesophageal cancer. $\mathrm{Br} J$ Cancer 94: $637-641$

Crumley AB, Stuart RC, McKernan M, McDonald AC, McMillan DC (2008) Comparison of an inflammation-based prognostic score (GPS) with performance status (ECOG-ps) in patients receiving palliative chemotherapy for gastroesophageal cancer. J Gastroenterol Hepatol 23: e325-e329

Duffy BK, Gurm HS, Rajagopal V, Gupta R, Ellis SG, Bhatt DL (2006) Usefulness of an elevated neutrophil to lymphocyte ratio in predicting long-term mortality after percutaneous coronary intervention. $\mathrm{Am} \mathrm{J}$ Cardiol 97: 993 - 996

Forrest LM, McMillan DC, McArdle CS, Angerson WJ, Dunlop DJ (2003) Evaluation of cumulative prognostic scores based on the systemic inflammatory response in patients with inoperable non-small-cell lung cancer. Br J Cancer 89: 1028-1030

Galon J, Costes A, Sanchez-Cabo F, Kirilovsky A, Mlecnik B, Lagorce-Pagès C, Tosolini M, Camus M, Berger A, Wind P, Zinzindohoué F, Bruneval P, Cugnenc PH, Trajanoski Z, Fridman WH, Pagès F (2006) Type, density, and location of immune cells within human colorectal tumours predict clinical outcome. Science 313: 1960-1964

Halazun K, Hardy MA, Rana AA, Woodland DC, Luyten EJ, Mahadev S, WItkowski P, Stegel AB, Brown RS, Emond JC (2009) Negative impact of neutrophil lymphocyte ratio on outcome after liver transplantation for hepatocellular carcinoma. Ann Surg 250: 141 - 151

Halazun KJ, Aldoori A, Malik HZ, Al-Mukhtar A, Prasad KR, Toogood GJ, Lodge JP (2008) Elevated preoperative neutrophil to lymphocyte ratio predicts survival following hepatic resection for colorectal liver metastases. Eur J Surg Oncol 34: 55-60

Hartman M, Loy EY, Ku CS, Chia KS (2010) Molecular epidemiology and its current clinical use in cancer management. Lancet Oncol 11: $383-390$

Hilmy M, Bartlett JMS, Underwood MA, McMillan DC (2005) The relationship between the systemic inflammatory response and survival in patients with transitional cell carcinoma of the urinary bladder. Br J Cancer 92: 625-627

Ishizuka M, Nagata H, Takagi K, Kubota K (2009) Influence of inflammation-based prognostic score on mortality of patients undergoing chemotherapy for far advanced or recurrent unresectable colorectal cancer. Ann Surg 250: 268-272

Johnson TV, Abbasi A, Owen-Smith A, Young A, Ogan K, Pattaras J, Nieh P, Marshall FF, Master VA (2010) Absolute pre-operative C-reactive protein predicts metastasis and mortality in the first year following potentially curative nephrectomy for clear cell renal cell cancer. J Urol 183: $480-485$

Kishi Y, Kopetz S, Chun Y, Palavecino M, Abdalla E, Vauthey J-N (2009) Blood neutrophil-to-lymphocyte ratio predicts survival in patients with colorectal liver metastases treated with systemic chemotherapy. Ann Surg Oncol 16: 614-622

Koopman M, Venderbosch S, Nagtegaal ID, van Krieken JH, Punt CJ (2009) A review on the use of molecular markers of cytotoxic therapy for colorectal cancer, what have we learned? Eur J Cancer 45: 1935-1949 
Liu H, Liu G, Bao Q, Sun W, Bao H, Bi L, Wen W, Liu Y, Wang Z, Yin X, Bai Y, Hu X (2010) The Baseline Ratio of neutrophils to lymphocytes is associated with patient prognosis in rectal carcinoma. J Gastrointest Cancer 41: $116-120$

McMillan DC (2009) Systemic inflammation, nutritional status and survival in patients with cancer. Curr Opin Nutr Metab Care 12: 223-226

Michael M, Goldstein D, Clarke SJ, Milner AD, Beale P, Friedlander M, Mitchell P (2006) Prognostic factors predictive of response and survival to a modified FOLFOX regimen: importance of an increased neutrophil count. Clin Colorectal Cancer 6: 297-304

Mitsuru I, Junji K, Mitsugi S, Kyu R, Masato K, Tokihiko S (2009) Systemic inflammatory response predicts postoperative outcome in patients with liver metastases from colorectal cancer. J Surg Oncol 100: 38-42

Núñez J, Núñez E, Bodí V, Sanchis J, Miñana G, Mainar L, Santas E, Merlos P, Rumiz E, Darmofal H, Heatta AM, Llàcer A (2008) Usefulness of the neutrophil to lymphocyte ratio in predicting long-term mortality in ST segment elevation myocardial infarction. Am J Cardiol 101: $747-752$

Pagès F, Berger A, Camus M, Sanchez-Cabo F, Costes A, Molidor R, Mlecnik B, Kirilovsky A, Nilsson M, Damotte D, Meatchi T, Bruneval P, Cugnenc PH, Trajanoski Z, Fridman WH, Galon J (2005) Effector memory T cells, early metastasis and survival in CRC. $N$ Eng J Med 353: 2654-2666

Prado CMM, Lieffers JR, McCargar LJ, Reiman T, Sawyer MB, Martin L, Baracose VE (2008) Prevalence and clinical implications of sarcopenic obesity in patients with solid tumours of the respiratory and gastrointestinal tracts: a population-based study. Lancet Oncol 9: 629-635

Roxburgh CSD, McMillan DC (2010) Role of systemic inflammatory response in predicting survival in patients with primary operable cancer. Future Oncol 6: 149-163
Smith RA, Bosonnet L, Raraty M, Sutton R, Neoptolemos JP, Campbell F, Ghaneh P (2009) Preoperative platelet-lymphocyte ratio is an independent significant prognostic marker in resected pancreatic ductal adenocarcinoma. Am J Surg 197: 466-472

Subramanian J, Simon R (2010) Gene expression-based prognostic signatures in lung cancer: ready for clinical use? J Natl Cancer Inst 102: $464-474$

Teramukai S, Kitano T, Kishida Y, Kawahara M, Kubota K, Komuta K, Minato K, Mio T, Fujita Y, Yonei T, Nakano K, Tsuboi M, Shibata K, Furuse K, Fukushima M (2009) Pretreatment neutrophil count as an independent prognostic factor in advanced non-small-cell lung cancer: an analysis of Japan Multinational Trial Organisation LC00-03. Eur J Cancer 45: $1950-1958$

Van Cutsem E, Köhne CH, Hitre E, Zaluski J, Chang Chien CR, Makhson A, D’Haens G, Pintér T, Lim R, Bodoky G, Roh JK, Folprecht G, Ruff P, Stroh C, Tejpar S, Schlichting M, Nippgen J, Rougier P (2009) Cetuximab and chemotherapy as initial treatment for metastatic colorectal cancer. $N$ Eng J Med 360: 1408-1417

Wacholder S, Hartge P, Prentice R, Garcia-Closas M, Feigelson HS, Diver WR, Thun MJ, Cox DG, Hankinson SE, Kraft P, Rosner B, Berg CD, Brinton LA, Lissowska J, Sherman ME, Chlebowski R, Kooperberg C, Jackson RD, Buckman DW, Hui P, Pfeiffer R, Jacobs KB, Thomas GD, Hoover RN, Gail MH, Chanock SJ, Hunter DJ (2010) Performance of common genetic variants in breast-cancer risk models. $N$ Eng J Med 362: 986-993

World Health Organisation (2004) Global burden of disease 2004. http:// www.who.int/healthinfo/global_burden_disease/2004_report_update/en

Yamanaka T, Matsumoto S, Teramukai S, Ishiwata R, Nagai Y, Fukushima M (2007) The baseline ratio of neutrophils to lymphocytes is associated with patient prognosis in advanced gastric cancer. Oncology 73: 215-220 